\title{
Artigos
}

\section{A INFLUÊNCIA DA EMOÇÃO SURPRESA NA DECISÃO DE RECOMPRA DE PRODUTOS DE MODA SUSTENTÁVEL}

DOI: 10.19177/rgsa.v9e0I202044-64

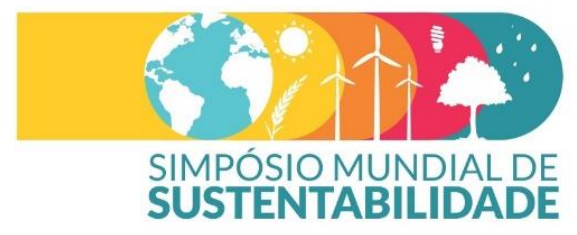

\author{
Luana Poletto Barbieri ${ }^{1}$ \\ Igor Bosa² \\ Janine Fleith de Medeiros ${ }^{3}$ \\ Cassiana Maris Lima $\mathrm{Cruz}^{4}$
}

\section{RESUMO}

O estudo das emoções mostra-se cada vez mais relevante para a compreensão do comportamento dos consumidores e sua jornada de compra. Diante disso, a presente pesquisa tem por objetivo investigar a influência das emoções no processo de decisão de compra de produtos ambientalmente sustentáveis, de modo a avaliar o impacto da surpresa sobre a intenção de recompra de produtos de moda sustentável. Uma série de estudos demonstram que a surpresa consiste em um determinante emocional para a satisfação, visto que pode intensificar tanto emoções positivas quanto negativas, aumentando ou reduzindo o nível de satisfação do consumidor. Para alcance dos objetivos, foi realizada uma pesquisa causal, quantitativa, aplicada ao público universitário. Os resultados indicam que as emoções, por si só, não são suficientes para mensurar a intenção de recompra de produtos de moda sustentável. Além disso, verificou-se que o efeito da emoção surpresa sobre a intenção de recompra aumenta quanto essa possui valência negativa. Finalmente, novos estudos são propostos,

\footnotetext{
${ }^{1}$ Graduada em Publicidade e Propaganda. Universidade de Passo Fundo. 142841@upf.br

${ }^{2}$ Graduado em Engenharia de Produção. Universidade de Passo Fundo.145676@upf.br

${ }^{3}$ Doutora em Engenharia de Produção. Universidade de Passo Fundo. Janine@upf.br

${ }^{4}$ Doutora em Comunicação Social. Universidade de Passo Fundo. Cassiana@upf.br
} 
ampliando-se o número de construtos para que se possa melhor compreender o processo de recompra para produtos de moda sustentável.

Palavras-chave: Comportamento do consumidor. Emoções. Surpresa. Moda sustentável.

\title{
THE INFLUENCE OF SURPRISE EMOTION ON THE DECISION TO BUYBACK SUSTAINABLE FASHION PRODUCTS
}

\begin{abstract}
The study of emotions shows increasingly relevant to the understanding of consumer behavior and their shopping journey. Given this, this research aims to investigate the influence of emotions in the decision-making process of buying sustainable environmental products, in order to evaluate the impact of surprise on the intention to recommend sustainable fashion products. A number of studies show that surprise is an emotional determinant for satisfaction, as it can intensify both positive and negative emotions, increasing or reducing the level of consumer satisfaction. To reach the objectives, a causal, quantitative research was applied to the university public. The results make it possible to state that emotions alone are not sufficient to measure the buyback intention of sustainable fashion products. In addition, it was found that the effect of surprise emotion on repurchase intention increases when it has negative valence. Finally, new studies are proposed expanding the number of constructs to have better understand the buyback process for sustainable fashion products.
\end{abstract}

Keywords: Consumer behavior. Emotions. Surprise. Sustainable fashion.

\section{INTRODUÇÃO}

O estudo das emoções mostra-se cada vez mais relevante para a compreensão do comportamento e da jornada de compra do consumidor, visto que o consumo passou a ser visto como um fluxo de fantasias e sentimentos, assim caracterizandose como um estado essencialmente subjetivo, composto de uma vasta gama de significados simbólicos, respostas hedônicas e critérios estéticos (HOLBROOK; HIRSCHMAN, 1982). Neste cenário, uma série de pesquisas tem investigado o papel 
das emoções na satisfação do consumidor (WESTBROOK; OLIVER, 1991; JOHNSON; ANDERSON; FORNELL, 1995; ALMEIDA, 2003; LARÁN; ROSSI, 2006; MONTELONGO, 2016).

Dentre as emoções, a surpresa destaca-se como um determinante emocional para a satisfação, visto que essa pode intensificar tanto emoções positivas, propiciando maiores níveis de satisfação, quanto emoções negativas, resultando em um impacto negativo nos níveis de satisfação (LARÁN; ROSSI, 2006). No que tange a surpresa negativa, Kieling, Brei e Vieira (2016) destacam que a experiência da surpresa negativa em um processo de compra condiciona o consumidor a acreditar no desempenho inferior da oferta, impulsionando o mesmo a buscar marcas alternativas.

Embora o número de trabalhos que focaram na emoção surpresa seja considerável (LARÁN; ROSSI, 2006; EBERT; MEYVIS, 2014; MONTELONGO, 2014; KIELING; BREI; VIEIRA, 2016), percebe-se que maioria prioriza a compra inicial em detrimento da compra repetida (BLACKWELL; MINIARD; ENGEL, 2005;). Em vista disso, a presente pesquisa tem por objetivo investigar a influência da emoção surpresa no processo de decisão de recompra de produtos ambientalmente sustentáveis.

Tais produtos podem ser definidos como produtos que exploram os recursos naturais de forma equilibrada, propiciando alta qualidade social, mínimo desperdício e danos a natureza, contribuindo assim tanto com a sociedade, quanto com o meio ambiente (MANZINI; VEZZOLI, 2002). A escolha de produtos sustentáveis se deu em função do crescente número de pesquisas referentes a consumo sustentável nos últimos anos (REFOSCO; et al., 2011; FERRAZ; et al., 2013; BROUGH; et al., 2016; MÜLLER, 2016), fato esse que torna pertinente a análise do comportamento de consumidores de tal categoria de produtos.

A presente pesquisa direcionou seu foco para o setor de moda, visto que este é um dos setores mais difundidos espacialmente, caracterizando-se como uma notável fonte de empregos e geração de renda, sendo a terceira atividade econômica em geração de renda e movimentação financeira (LUPATINI, 2004; BERLIM, 2012). Todavia, após sua notável expansão, ocorrida nas duas últimas décadas, consolidouse, no setor, uma abordagem caracterizada pela produção de peças homogêneas destinadas a rápida aquisição e descarte (FLETCHER, 2010). Tal estímulo ao consumo de peças de vestuário facilmente substituíveis representa uma desvantagem 
significativa em termos de sustentabilidade ambiental e social (TODESCHINI; et al.,2017), dado o aumento do volume de resíduos têxteis (DISSANAYAKE; SINHA, 2012). Em vista disso, a preocupação com a sustentabilidade ambiental ganhou destaque no universo da Moda (SCHULTE; LOPEZ, 2007), assim tornando pertinente a escolha deste setor como objeto de estudo da presente pesquisa.

\section{FUNDAMENTAÇÃO TEÓRICA}

\subsection{Decisão de compra}

$\mathrm{O}$ ato de compra é resultante de um processo decisório (BLACKWELL; MINIARD; ENGEL, 2005), o qual pode ser compreendido como um processo de demanda estratégica, visto que é necessário realizar uma avaliação do esforço dedicado à escolha (SOLOMON, 2016). Em vista disso, observa-se que a compra não apresenta características de um ato de pura espontaneidade, mas sim de um processo psicológico de conflitos internos (RICHERS, 1984), cabendo ao estudo do comportamento do consumidor compreender as razões que levam o indivíduo a realizar tal ato (SCHIFFMAN; KANUK, 2000; KARSAKLIAN, 2009).

O processo de decisão de compra pode ocorrer de diferentes maneiras, podendo se caracterizar como uma compra de maior envolvimento, demandando maior abrangência na busca de informações e dedicação para a avaliação de alternativas pré-compra (SAMARA; MORSCH, 2005), ou então como uma compra de baixo envolvimento, demandando menor planejamento e muitas vezes tomadas de decisão no próprio ponto de venda (ETZEL; WALKER; STANTON, 2001).

De acordo com Assael (1992), os diferentes tipos de comportamentos de compra podem ser divididos em quatro categorias: (i) compra complexa; (ii) compra por dissonância reduzida; (iii) compra habitual; e (iv) compras por busca de variedade.

Nas (i) compras complexas os consumidores conhecem as marcas e sabem diferenciá-las, apresentando elevado grau de envolvido. Usualmente esse tipo de comportamento ocorre em compras de produtos que apresentam alto valor envolvido, e cuja frequência de aquisição é baixa. Em (ii) compras por dissonância reduzida os consumidores percebem poucas diferenças entre as marcas, embora estejam envolvidos e realizando pesquisa em pontos de vendas, efetuam a tomada de decisão

R. gest. sust. ambient., Florianópolis, v. 9, n. esp, p. 44-64, mai. 2020. 
com maior agilidade do que nas compras complexas. Com relação às (iii) compras habituais, nestas os consumidores não diferem as marca, apresentam baixo envolvimento, não realizam pesquisa e, na ausência do produto procurado, optam por outras marcas. Finalmente, nas (iv) compras por busca de variedade, os compradores percebem acentuadas diferenças entre as marcas disponíveis, mas por estarem pouco envolvidos com a compra, buscam por novos ofertantes em decorrência da quantidade de ofertas disponíveis e da ansiedade por vivenciar outras opções.

$\mathrm{O}$ ato de compra pode ser classificado como compra inicial, voltada à solução de problemas restritos ou como compra repetida, voltada à solução de problemas repetidos (BLACKWELL; MINIARD; ENGEL, 2005). Cabe destacar que o processo decisório do consumidor pode ser influenciada pelo fato de a compra ser inicial ou repetida (SCHIFFMAN; KANUK, 2000), visto que, de acordo com Simonson e Tversky (1992), as preferências são sistematicamente influenciadas pelas compensações e pelas opções anteriores.

De acordo com Blackwell, Miniard e Engel (2005), os indivíduos costumam ser racionais no processo de decisão, considerando as implicações de suas ações antes de concretizar o ato da compra por meio de três fatores determinantes: (i) diferenças individuais (como demografia, psicografia, valores, personalidade, recursos econômicos, motivação, conhecimento e atitudes); (ii) influências ambientais (advindas de sua cultura, classe social, família, influência pessoal e situação); e (iii) processos psicológicos (como processos da informação, aprendizagem, mudança de comportamentos e atitudes). De acordo com Sheth, Mittal e Newman (2001), Blackwell, Miniard e Engel (2005) e Solomon (2016), o processo de decisão de compra pode ser estruturado de forma geral, conforme apresentado na Figura 1.

Figura 1: Estágios de Processo Decisório de Compra (PDC)

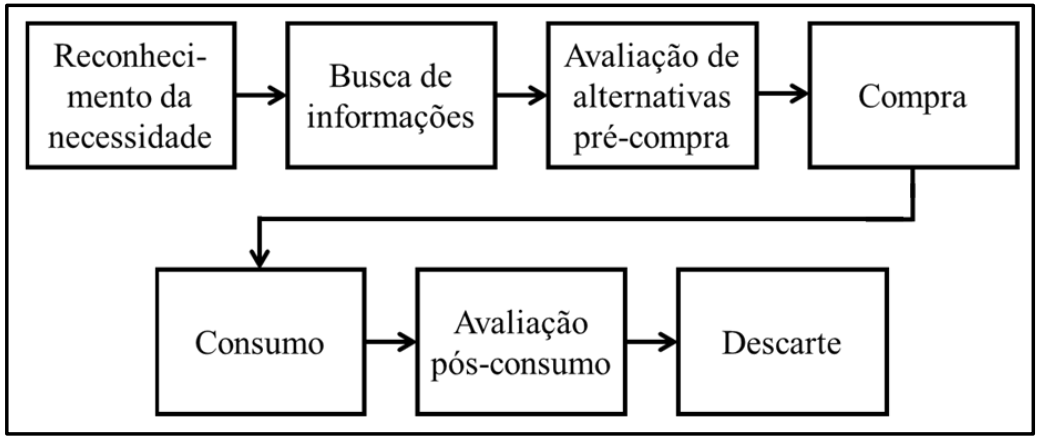

Fonte: Autores (2019), adaptado de Blackwell, Miniard e Engel (2005).

R. gest. sust. ambient., Florianópolis, v. 9, n. esp, p. 44-64, mai. 2020. 
O modelo de processo proposto pelos autores tem início com o reconhecimento da necessidade (BLACKWELL; MINIARD; ENGEL, 2005; CHURCHILL; PETER, 2012; KOTLER; ARMSTRONG, 2015). Posteriormente, tem-se a etapa de busca de informações (SHETH; MITTAL; NEWMAN, 2001; BLACKWELL; MINIARD; ENGEL, 2005; SOLOMON, 2016), a qual pode ser realizada tanto em fontes internas, (memória, tendências genéticas ou situações vivenciadas), quanto em fontes externas (grupos e fontes comerciais) (BLACKWELL; MINIARD; ENGEL, 2005; KOTLER; KELLER, 2012).

A partir das informações levantadas na etapa "busca de informações" o consumidor realiza a avaliação de alternativas pré-compra. Nessa etapa o cliente já possui as informações necessárias, passando à avaliar as alternativas a partir de critérios de seleção que visam possibilitar a escolha da opção que melhor atenda suas necessidades, desejos e restrições (SHETH; MITTAL; NEWMAN, 2001; BLACKWELL; MINIARD; ENGEL, 2005; SOLOMON, 2016).

Após a avaliação das alternativas, o consumidor decide pela compra (SHETH; MITTAL; NEWMAN, 2001; SOLOMON, 2016). Esta etapa do processo pode parecer um passo direito, mas o consumidor ainda possui alternativas de escolha, visto que 0 mesmo passa por outras fases de escolha, dentre elas a escolha de canal de venda (loja física, online, catálogo), e estando sujeito à interferências (vendedor, vitrine, propaganda) quando opta por realizar a compra no interior da loja (BLACKWELL; MINIARD; ENGEL, 2005). Além disso, uma série de fatores, como a demora ou indisponibilidade do produto e infidelidade com a propaganda, podem vir a influenciar o consumidor a alterar sua decisão de compra (KOTLER; KELLER, 2012).

Após a compra ocorre a etapa de consumo, na qual o consumidor utiliza o produto e vivencia a experiência após a realização da compra (SOLOMON, 2016). Tal experiência de uso fornece informações e memórias que influenciarão o consumidor na realização de uma possível próxima compra (SHETH; MITTAL; NEWMAN, 2001).

Por fim, após ter vivenciado a experiência de consumo, o comprador adquire uma opinião referente ao desempenho do produto (KOTLER; KELLER, 2012; SOLOMON, 2016). As sensações decorrentes podem gerar a satisfação, que ocorre quando as expectativas são atendidas, ou a insatisfação, que ocorre quando as experiências não atendem ao esperado. Finalmente, a última etapa do processo 
relaciona-se ao descarte, que pode se dar em forma de: (I) Descarte completo; (II) Reciclagem; ou (III) Revenda (BLACKWELL; MINIARD; ENGEL, 2005).

\subsection{Emoções}

As emoções são estados mentais de prontidão que surgem da avaliação de eventos ou dos próprios pensamentos (BAGOZZI; GOPINATH; NYER, 1999). Segundo a teoria de James-Lange, baseiam-se no reconhecimento dos sentimentos decorrentes de estados físicos de ativação. São, portanto, estados decorrentes de distintas situações, manifestando-se por meio de alterações fisiológicas, como aumento da frequência cardíaca, expressões faciais, entre outros (FADIMAN; FRAGER 2008).

De acordo com Sheth, Mittal e Newman (2001), as emoções podem ser descritas como "consequência da ocorrência de alguma excitação fisiológica seguida por uma resposta comportamental". Assim, os autores as dividem em três componentes: (i) fisiológico (estímulo recebido pelo corpo); (ii) cognitivo (interpretação do estímulo recebido); e (iii) comportamental (ação realizada a partir da interpretação do estímulo).

Ao longo do tempo, uma série de estudos referentes às emoções e sua influência têm sido realizados. Neste sentido, destaca-se o estudo de Sheth, Mittal e Newman (2001), que apresenta a Teoria Bifatorial de Schachter, a qual afirma que a emoção depende dos fatores de "excitação autônoma", proveniente de estímulos externos e internos, sendo a emoção um resultado da interação de tais estímulos (Figura 2), caracterizando a emoção como uma reação do corpo, a qual ocorreria somente após o estímulo, manifestando-se em forma de mudança fisiológica (FADIMAN; FRAGER, 2008). 
Figura 2: Modelo de Processo Emocional

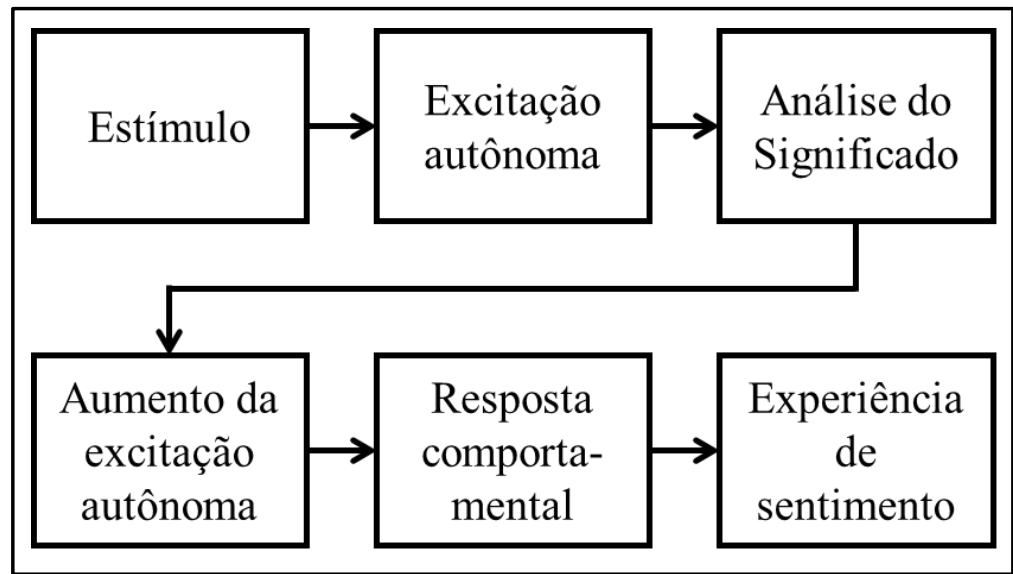

Fonte: Autores (2019), adaptado de Sheth, Mittal e Newman (2001).

Adicionalmente, distintos pesquisadores desenvolveram escalas para analisar as emoções. Em 1980 o psicólogo Robert Plutchik identificou oito emoções primárias, as quais podem apresentar variações em sua intensidade, sendo as demais emoções provenientes da combinação de duas ou mais emoções primárias (Figura 3) (SHETH; MITTAL; NEWMAN, 2001). As emoções primárias mapeadas no estudo foram: (i) medo; (ii) raiva; (iii) alegria; (iv) tristeza; (v) aceitação; (vi) aversão; (vii) antecipação; e (viii) surpresa.

Figura 3: Tipologia das Emoções: círculo de Plutchik

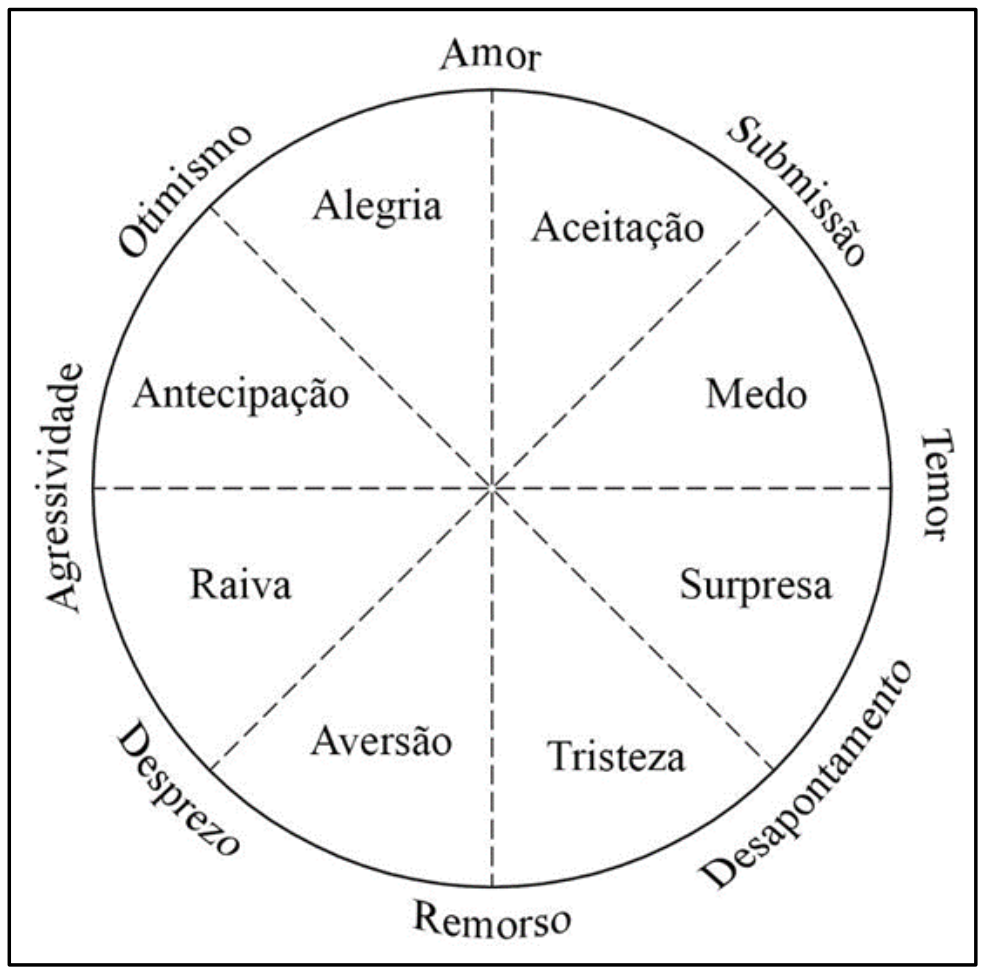

Fonte: Autores (2019), adaptado Sheth, Mittal e Newman (2001)

R. gest. sust. ambient., Florianópolis, v. 9, n. esp, p. 44-64, mai. 2020. 
As emoções desempenham um papel fundamental na decisão do consumidor (LARÁN; ROSSI, 2006). No que tange à relação entre as emoções e o consumo, cabe destacar a escala CES (Consumption Emotions Set). Desenvolvida por Richins (1997) tendo como foco o comportamento de compra, é composta por 47 descritores distribuídos em 17 fatores. A escala CES possibilita a medição das emoções que se manifestam ao longo da compra e do consumo (LAROS; STEENKAMP, 2005), abrangendo tanto emoções positivas quanto emoções negativas (Tabela 1).

Tabela 1: Escala CES, Fatores e Descritivos

\begin{tabular}{|c|c|c|c|}
\hline Fatores & Descritores & Fatores & Descritores \\
\hline Raiva & $\begin{array}{l}\text { - Frustrado } \\
\text { - Irado } \\
\text { - Irritado }\end{array}$ & Amor & $\begin{array}{l}\text { - Amoroso } \\
\text { - Sentimental } \\
\text { - Afetuoso }\end{array}$ \\
\hline Descontentamento & $\begin{array}{l}\text { - Insatisfeito } \\
\text { - Descontente }\end{array}$ & Tranquilidade & $\begin{array}{l}\text { - Calmo } \\
\text { - Em paz }\end{array}$ \\
\hline Preocupação & $\begin{array}{l}\text { - Nervoso } \\
\text { - Preocupado } \\
\text { - Tenso }\end{array}$ & Contentamento & $\begin{array}{l}\text { - Contente } \\
\text { - Realizado }\end{array}$ \\
\hline Tristeza & $\begin{array}{l}\text { - Depressivo } \\
\text { - Triste } \\
\text { - Extremamente infeliz }\end{array}$ & Otimismo & $\begin{array}{l}\text { - Otimista } \\
\text { - Encorajado } \\
\text { - Esperançoso }\end{array}$ \\
\hline Medo & $\begin{array}{l}\text { - Assustado } \\
\text { - Com medo } \\
\text { - Em pânico }\end{array}$ & Alegria & $\begin{array}{l}\text { - Feliz } \\
\text { - Satisfeito } \\
\text { - Alegre }\end{array}$ \\
\hline Vergonha & $\begin{array}{l}\text { - Envergonhado } \\
\text { - Constrangido } \\
\text { - Humilhado }\end{array}$ & Animação & $\begin{array}{l}\text { - Animado } \\
\text { - Extasiado } \\
\text { - Entusiasmado }\end{array}$ \\
\hline Inveja & $\begin{array}{l}\text { - Invejoso } \\
\text { - Com ciúmes }\end{array}$ & Surpresa & $\begin{array}{l}\text { - Surpreso } \\
\text { - Admirado } \\
\text { - Atônito }\end{array}$ \\
\hline Solidão & $\begin{array}{l}\text { - Solitário } \\
\text { - Com saudades de casa }\end{array}$ & Outros itens & $\begin{array}{l}\text { - Culpado } \\
\text { - Orgulhoso } \\
\text { - Ansioso } \\
\text { - Aliviado }\end{array}$ \\
\hline Amor Romântico & $\begin{array}{l}\text { - Sexy } \\
\text { - Romântico } \\
\text { - Apaixonado }\end{array}$ & & \\
\hline
\end{tabular}

Muitas questões que abrangem o campo de estudo das emoções continuam sem solução (RICHINS, 1997), destacando-se a relação existente entre a emoção R. gest. sust. ambient., Florianópolis, v. 9, n. esp, p. 44-64, mai. 2020. 
surpresa e satisfação, retenção, lealdade e insatisfação do consumidor (ROSSI; SLONGO, 1997). De fato, embora as emoções sejam constante alvo de pesquisas no campo da psicologia, figuram em um número reduzido de publicações dedicadas a compreensão de sua relação com o comportamento do consumidor (LARÁN; ROSSI, 2006).

Focando na emoção surpresa, a mesma pode ser considerada uma emoção devido a sua espontaneidade e duração limitada, sendo uma emoção de curto prazo, normalmente associada a outra emoção. Portanto, pode-se dizer que a surpresa por si só possui valência neutra, caracterizando-se como positiva quando associada a uma emoção positiva, e consequentemente caracterizando-se como negativa quando associada a uma emoção negativa (MONTELONGO, 2014).

A ligação entre surpresa e satisfação parece se materializar através de um processo do tipo "afeto como informação", de modo que a surpresa, pode impactar diretamente e indiretamente, por meio de emoções positivas ou negativas, a satisfação do consumidor, sendo tais emoções apenas vetores da influência da surpresa na satisfação (VANHAMME, 2001).

A surpresa pode ocorrer em casos de superestimação ou subestimação, podendo estar associada a reações positivas ou negativas (BLACK; GREGERSEN, 1990), visto que a essa pode estar associada tanto a emoções positivas quanto a emoções negativas (WESTBROOK; OLIVER, 1991).

De modo geral, conforme apontado por Larán e Rossi (2006), a surpresa positiva gera emoções de caráter positivo influenciando positivamente a satisfação, além de influenciar negativamente possíveis emoções negativas relativas à experiência de consumo. Já a surpresa negativa apresenta um impacto semelhante, mas no sentido contrário, amplificando emoções negativas e impactando negativamente na satisfação.

\section{MÉTODO}

Para atender ao objetivo proposto pelo estudo, realizou-se inicialmente uma pesquisa bibliográfica, narrativa, seguida da realização de uma pesquisa causal. Pesquisas causais devem ser empregadas quando se busca obter evidências da 
existência de uma relações de causalidade entre variáveis, de modo a determinar a natureza da relação entre as variáveis causais e o efeito a ser previsto (MALHOTRA, 2012). Além disso, optou-se pela abordagem quantitativa, uma vez que esta propõe ir além da simples identificação da relação entre variáveis, auxiliando na determinação da natureza dessa relação (GIL, 1999), assim possibilitando o alcance de resultados conclusivos (MALHOTRA, 2012).

Diante do exposto, e visando conhecer o efeito da surpresa positiva e da surpresa negativa na intenção de recompra de produtos de moda sustentável, foram elaborados cenários hipotéticos. O Cenário 1 objetivava desencadear emoções positivas, enquanto o Cenário 2 focava em emoções negativas. Cada um dos cenários foi composto por duas situações distintas, sendo a primeira relacionada a um fator de sustentabilidade (Situação $A$ ), e a segunda relacionada à qualidade do produto (Situação B) conforme apresentado no Quadro 1.

\section{Quadro 1: Cenários Elaborados}

\begin{tabular}{|c|c|c|}
\hline & Sustentabilidade (Situação A) & Qualidade (situação B) \\
\hline 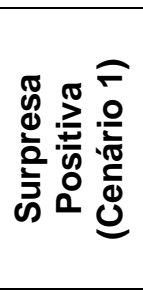 & $\begin{array}{l}\text { Alguns dias após a realização da } \\
\text { compra chegaria a seu conhecimento } \\
\text { que a linha de produtos havia } \\
\text { recebido o Prêmio Mundial de } \\
\text { Sustentabilidade, concedido pela } \\
\text { World Packaging Organization } \\
\text { (WPO), }\end{array}$ & $\begin{array}{l}\text { Após ser submetido à primeira lavagem o } \\
\text { produto manteve todas as suas } \\
\text { características, e que após alguns dias } \\
\text { receberam um desconto de } 50 \% \text { para } \\
\text { futuras compras desta mesma linha. }\end{array}$ \\
\hline 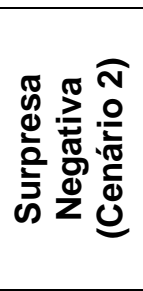 & $\begin{array}{l}\text { Alguns dias após a realização da } \\
\text { compra, foi descoberto que a linha } \\
\text { era uma fraude, de forma que o } \\
\text { produto não poderia ser reciclado e } \\
\text { nem se degradaria com o passar dos } \\
\text { anos, assim causando danos ao meio } \\
\text { ambiente. }\end{array}$ & $\begin{array}{l}\text { Após ser submetido à primeira lavagem o } \\
\text { produto sofreu alterações em suas } \\
\text { características, não sendo possível } \\
\text { realizar a troca ou a reposição da peça } \\
\text { junto à loja }\end{array}$ \\
\hline
\end{tabular}

Fonte: Autores (2019)

O procedimento de coleta de dados se deu por meio da aplicação de dois questionários, sendo o primeiro destinado a mensuração das emoções em uma situação de surpresa positiva (Cenário 1) e o segundo destinado a mensuração das emoções em uma situação de surpresa negativa (Cenário 2). Os instrumentos utilizados fizeram uso de questões com escala Likert, criadas no intuito de mensurar as atitudes e as emoções dos alunos com base em uma escala de intervalos. A amostra utilizada foi composta por 60 estudantes de uma universidade situada na região norte do estado do Rio Grande do Sul, os quais 
foram igualmente distribuídos entre os questionários referentes aos cenários apresentados, não sendo feitas distinções por curso e por nível.

Ao iniciarem o questionário, os participantes foram introduzidos ao tema moda sustentável, sendo posteriormente apresentados à uma linha de produtos sustentáveis. Na sequência, os participantes tiveram acesso a mesma imagem que apresentava os produtos, sendo em seguida apresentados aos cenários hipotéticos elaborados. Por fim, os participantes foram direcionados à uma série de questões elaboradas para mensurar as emoções dos consumidores em cada uma das experiências de consumo apresentadas.

A análise dos dados coletados foi realizada por meio de modelos de regressão linear simples (RLS), visto que esta possibilita a verificação da relação entre uma variável explicativa métrica (variável independente) e uma variável independente métrica, propiciando a análise do impacto de cada variável explicativa e a efetividade do modelo (FAVERO; et al., 2009).

\section{RESULTADOS E DISCUSSÃO}

Esta seção apresenta os resultados da pesquisa, abordando inicialmente os (i) resultados referentes ao impacto da surpresa positiva (Cenário 1); e posteriormente os (ii) resultados referentes ao impacto da surpresa negativa (Cenário 2).

\subsection{Resultados Grupo Surpresa Positiva}

A partir dos dados coletados foram realizadas análises de RLS, no intuito de avaliar o impacto da variável independente (VI) surpresa positiva (SP) sobre as variáveis dependentes (VD) recompra da marca $(\mathrm{RM})$ e recompra da linha de produtos $(R L)$, avaliando a significância do modelo através do valor-p (Sig.). Os modelos desenvolvidos (Quadro 2) avaliam o comportamento das variáveis supracitadas em ambas as situações, sustentabilidade $(A)$ e qualidade $(B)$ no cenário de surpresa positiva (Cenário 1). 
Quadro 2: Modelos RLS Cenário 1

\begin{tabular}{|c|c|c|c|c|c|c|c|}
\hline \multirow{2}{*}{ Modelo } & \multirow{2}{*}{ Situação } & \multicolumn{2}{|c|}{ Descrição } & \multirow{2}{*}{ R } & $\mathbf{R}^{2}$ & F & Sig. \\
\cline { 3 - 7 } & & VD & VI & & & & \\
\hline 1 & $\mathrm{~A}$ & $\mathrm{RM}$ & $\mathrm{SP}$ & 0.230 & 0.053 & 1.569 & 0.211 \\
\hline 2 & $\mathrm{~A}$ & $\mathrm{RL}$ & $\mathrm{SP}$ & 0.304 & 0.092 & 2.852 & 0.102 \\
\hline 3 & $\mathrm{~B}$ & $\mathrm{RM}$ & $\mathrm{SP}$ & 0.125 & 0.016 & 0.442 & 0.512 \\
\hline 4 & $\mathrm{~B}$ & $\mathrm{RL}$ & $\mathrm{SP}$ & 0.021 & 0.000 & 0.012 & 0.912 \\
\hline
\end{tabular}

Fonte: Autores (2019)

Os modelos desenvolvidos para este cenário apontam que a VI surpresa positiva não é capaz de explicar satisfatoriamente o comportamento das VDs recompra da marca e recompra da linha, visto que os modelos apresentam um valorp de 0,211 (Modelo 1); 0,102 (Modelo 2); 0,512 (Modelo 3); e 0,912 (Modelo 4), estando todos acima do valor-p de 0,05 estabelecido para um intervalo de confiança de $95 \%$. Em vista disso, pode-se observar que, por mais que o consumidor seja surpreendido positivamente, essa surpresa não o faz querer realizar uma nova compra do produto, sendo este comportamento melhor explicado por outras variáveis independentes não abordadas no modelo de RLS.

Tal efeito pode ser atribuído ao fato de que, por mais que a surpresa positiva acarrete níveis de satisfação superiores provenientes da intensificação das emoções positivas (LARÁN; ROSSI, 2006), essa por si só não é suficiente para gerar a intenção de recompra do produto. Talvez isso se justifique em função de que os consumidores jovens considerem a compra de vestuário como sendo habitual, ou seja, de alta frequência e baixo envolvimento (ASSAEL, 1992).

\subsection{Resultados Grupo Surpresa Negativa}

A partir dos dados coletados foram realizadas análises de RLS, no intuito de avaliar o impacto da variável independente ( $\mathrm{VI})$ surpresa negativa (SN) sobre as variáveis dependentes (VD) recompra da marca $(R M)$ e recompra da linha de produtos $(R L)$, avaliando a significância do modelo através do valor-p (Sig.). Os modelos 
desenvolvidos (Quadro 3) avaliam o comportamento das variáveis supracitadas em ambas as situações, sustentabilidade (A) e qualidade (B) no cenário de surpresa negativa (Cenário 2).

Quadro 3: Modelos RLS Cenário 2

\begin{tabular}{|c|c|c|c|c|c|c|c|}
\hline \multirow{2}{*}{ Modelo } & \multirow{2}{*}{ Situação } & \multicolumn{2}{|c|}{ Descrição } & \multirow{2}{*}{$\mathbf{R}$} & $\mathbf{R}^{2}$ & F & Sig. \\
\cline { 3 - 5 } & & VD & VI & & & & \\
\hline 1 & $\mathrm{~A}$ & $\mathrm{RM}$ & $\mathrm{SN}$ & 0.333 & 0.111 & 3.491 & 0.072 \\
\hline 2 & $\mathrm{~A}$ & $\mathrm{RL}$ & $\mathrm{SN}$ & 0.367 & 0.135 & 4.365 & 0.046 \\
\hline 3 & $\mathrm{~B}$ & $\mathrm{RM}$ & $\mathrm{SN}$ & 0.542 & 0.293 & 11.624 & 0.002 \\
\hline 4 & $\mathrm{~B}$ & $\mathrm{RL}$ & $\mathrm{SN}$ & 0.210 & 0.044 & 1.288 & 0.266 \\
\hline
\end{tabular}

Fonte: Autores (2019)

Os modelos desenvolvidos para este cenário apontam que a VI surpresa negativa, em alguns casos, não é capaz de explicar satisfatoriamente o comportamento das VDs recompra da marca e recompra da linha, visto que o Modelo 1 e o Modelo 4 apresentam um valor-p de 0,072 (Modelo 1); e 0,266 (Modelo 4), os quais superam o valor-p de 0,05 estabelecido para um intervalo de confiança de $95 \%$. Em vista disso, pode-se observar que a surpresa negativa não afeta o comportamento de recompra da marca em uma situação onde as expectativas referentes a sustentabilidade são atendidas (Modelo 1), bem como não afeta a recompra de produtos da mesma linha em uma situação onde as expectativas referentes qualidade não são atendidas (Modelo 4).

Todavia, constatou-se que a surpresa negativa afeta a intenção de recompra da linha em uma situação de não atendimento das expectativas referentes a sustentabilidade (Modelo 2), bem como em uma situação de não atendimento das expectativas referentes a qualidade do produto (Modelo 3), os quais apresentam respectivamente um valor-p de 0,046 e 0,002, ambos inferiores ao valor-p 0,05 estabelecido para um intervalo de confiança de $95 \%$. Dessa forma, pode-se observar a forma como a surpresa negativa afeta a recompra do produto em ambos os casos, por meio da análise dos coeficientes no Quadro 4 e no Quadro 5, respectivamente. 
Quadro 4: Coeficientes Modelo 2

\begin{tabular}{|c|c|c|c|c|c|c|c|c|}
\hline & \multirow{2}{*}{ Modelo } & \multicolumn{2}{|c|}{$\begin{array}{l}\text { Coeficientes não } \\
\text { padronizados }\end{array}$} & \multirow{2}{*}{$\begin{array}{c}\begin{array}{c}\text { Coeficientes } \\
\text { Padronizados }\end{array} \\
\text { Beta }\end{array}$} & \multirow{2}{*}{$t$} & \multirow{2}{*}{ Sig. } & \multicolumn{2}{|c|}{ I.C. $95 \%$ para B } \\
\hline & & B & $\begin{array}{l}\text { Modelo } \\
\text { Padrão }\end{array}$ & & & & L. Inf. & L. Sup. \\
\hline \multirow{2}{*}{2} & (Constante) & 1.948 & 0.353 & - & 6.921 & 0.000 & 1.348 & 2.548 \\
\hline & Surpresa & -0.174 & 0.083 & -0.367 & -2.089 & 0.046 & -0.316 & -0.032 \\
\hline
\end{tabular}

Fonte: Autores (2019)

Tendo em vista os resultados apresentados no Quadro 4, pode-se observar que a surpresa afeta negativamente a decisão de recompra de produtos da mesma linha de produtos. Tal relação é expressa pela equação:

$$
\mathrm{RM}=\underset{(6.921)}{1.948}-\underset{(-2.089)}{0.174}(\mathrm{SN})+\mathrm{e}
$$

\begin{tabular}{|c|c|c|c|c|c|c|c|c|}
\hline & \multirow{2}{*}{ Modelo } & \multicolumn{2}{|c|}{$\begin{array}{c}\text { Coeficientes não } \\
\text { padronizados }\end{array}$} & \multirow{2}{*}{$\begin{array}{c}\begin{array}{c}\text { Coeficientes } \\
\text { Padronizados }\end{array} \\
\text { Beta }\end{array}$} & \multirow{2}{*}{$\mathbf{t}$} & \multirow{2}{*}{ Sig. } & \multicolumn{2}{|c|}{ I.C. $95 \%$ para B } \\
\hline & & B & $\begin{array}{l}\text { Modelo } \\
\text { Padrão }\end{array}$ & & & & L. Inf. & L. Sup. \\
\hline \multirow{2}{*}{3} & (Constante) & 3.643 & 0.526 & - & 5.519 & 0.000 & 2.747 & 4.538 \\
\hline & Surpresa & -0.429 & 0.126 & -0.542 & -3.409 & 0.002 & -0.640 & -0.215 \\
\hline
\end{tabular}

Quadro 5: Coeficientes Modelo 3

Fonte: Autores (2019)

A partir dos resultados apresentados no Quadro 5, pode-se observar que a surpresa negativa afeta negativamente a decisão de recompra de produtos da respectiva marca. Tal relação é expressa pela equação:

$$
\mathrm{RM}=\underset{(5.519)}{3.643}-\underset{(-3.409)}{0.429}(\mathrm{SN})+\mathrm{e}
$$

Dessa forma, pode-se observar que o não atendimento das expectativas geradas em função de um fator referente a sustentabilidade tende a condicionar 0

R. gest. sust. ambient., Florianópolis, v. 9, n. esp, p. 44-64, mai. 2020. 
consumidor a continuar consumindo produtos da marca, todavia evitando consumir produtos que integrem a linha causadora da surpresa negativa. Por outro lado, surpresas negativas referentes a qualidade do produto tendem a condicionar os consumidores a evitar a recompra de produtos da marca no geral e não somente da linha de produtos, constatando-se assim que, conforme apontado por Kotler e Keller (2012), a infidelidade ao produto pode decorrer de uma propaganda cujas expectativas levantadas não são adequadamente atendidas, influenciando o consumidor a alterar sua decisão de compra.

De modo geral, verificou-se que, por mais que a compra apresente um valor reduzido e um baixo envolvimento do consumidor, caracterizando-se como compra habitual (ASSAEL, 1992; ETZEL; WALKER; STANTON, 2001), a surpresa negativa pode influenciar os consumidores a não realizarem a recompra do produto. Tal efeito pode ser atribuído à intensificação das emoções negativas proveniente da quebra de expectativas do cliente, gerando um maior nível de insatisfação (LARÁN; ROSSI, 2006), condicionando o consumidor a acreditar que obterá menos prazer em sua compra, aumentando as chances do mesmo buscar marcas alternativas (KIELING; BREI; VIEIRA, 2016).

\section{CONCLUSÃO}

Diante das análises realizadas, observou-se que a surpresa positiva não representa uma influência significativa no ato de recompra. Entretanto, quando se trata de surpresa negativa, constatou-se que a intenção de recompra sofre influência desta emoção. Portanto, conclui-se que a emoção surpresa negativa tende a ser mais determinante para influenciar a recompra, visto que, conforme evidenciado por Kieling, Brei e Vieira (2016), a surpresa negativa apresenta uma influência negativa no prazer de realizar compras no futuro, dessa forma, indo ao encontro com os estudo realizados por Baumeister, Bratslavsky e Finkenauer (2001), que afirmam que as emoções negativas geram maior impacto e consequências mais intensas.

A proposta desta pesquisa foi contribuir com os estudos sobre o papel da surpresa no processo de decisão de recompra de produtos sustentáveis. Verificou-se, 
de maneira geral, que as emoções não são suficientes para medir esta variável e que produtos de baixo envolvimento não sofrem tanta influência do fator surpresa.

Esta pesquisa apresenta limitações referentes ao tamanho e a diversidade da amostra, visto que esta foi composta apenas por um número reduzido de estudantes universitários. Além disso, outra limitação diz respeito a abordagem de uma única categoria de produtos, assim limitando a percepção do impacto da surpresa sobre a decisão de recompra, visto que esta categoria é caracterizada pelo baixo envolvimento do cliente e pelo valor reduzido das compras.

Finalmente, sugere-se a continuidade da pesquisa na área da emoção relacionada a experiências de pós-consumo, seja no processo de satisfação ou recompra. Estudos futuros podem incluir novas variáveis a estas análises, tais como lealdade da marca, propaganda "boca-a-boca", nível de envolvimento com o produto, valores pessoais e preço. Também pode-se expandir a amostra a fim de aumentar a confiabilidade das análises, ou ainda focar em uma pesquisa em profundidade, para compreender melhor esse consumidor, suas atitudes e intenções. Acredita-se que a aplicação desta mesma pesquisa com produtos de maior envolvimento pode trazer dados mais conclusivos.

\section{REFERÊNCIAS}

ALMEIDA, S. O., Avaliação pós-consumo: proposição de uma escala para mensuração do encantamento do cliente. Dissertação (Mestrado em administração) PPG - Escola de Administração, Universidade Federal do Rio Grande do Sul, Porto Alegre, 2003.

ASSAEL, H. Consumer Behavior and Marketing Action. 4 ed. Boston: PWS-KENT Publishing Company (1992). 
BAUMEISTER, R.F.; BRATSLAVSKY, E.; FINKENAUER, C. Bad is stronger than good. Review of General Psychology, v. 5, n. 4, p. 323-370, 2001.

BERLIM, L. Moda e sustentabilidade: uma reflexão necessária. São Paulo: Estação das Letras e Cores, 2012.

BLACKWELL, R.D., MINIARD, P.W. E ENGEL, J.F. Comportamento do Consumidor. 9 ed. São Paulo: Pioneira Thomson Learning, 2005.

BROUGH, A.R.; et al. Is eco-friendly unmanly? The green-feminine stereotype and its effect on sustainable consumption. Journal of Consumer Research, v. 43, v. 4, p. 567-582, 2016.

CAMARGO, P.C.J. Neuromarketing: A Nova Pesquisa de Comportamento do Consumidor, São Paulo: Atlas, 2013.

CHANDON, P.; MORWITZ, V.G.; REINARTZ, W.J. The short- and long-term effects of measuring intent to repurchase. Journal of Consumer Research, v. 31, n. 3, p. 566572, 2004.

CHURCHILL, G. A.; PETER, J. P. Marketing: criando valor para o cliente. São Paulo: Saraiva, 2012.

DISSANAYAKE, G; SINHA, P. Sustainable Waste Management Strategies in the Fashion Industry Sector. The International Journal of Environmental Sustainability, v. 8, n. 1, p.77-90, 2012.

EBERT, J.E.J.; MEYVIS, T. Reading fictional stories and winning delayed prizes: the surprising emotional impact of distant events. Journal of Consumer Research, v. 40, n. 2, p. 203-222, 2013.

ETZEL, M. J.; WALKER, B. J.; STANTON, W. J. Marketing. São Paulo: Makron Books, 2001.

FADIMAN, J.; FRAGER, R. Personalidade e Crescimento Pessoal, 5 ed. Porto Alegre: Artmed 2008.

FAVERO, L. P.; et al. Análise de dados: modelagem multivariada para tomada de decisão. Rio de Janeiro: Elsevier, 2009.

FERRAZ, S.B.; et al. Produtos verdes: um estudo sobre atitude, intenção e comportamento de compra de universitários brasileiros. Revista de Administração UFSM, v. 9, n. 4, p. 605-623, 2016.

GIL, A.C. Métodos e técnicas de pesquisa social. São Paulo: Atlas, 1999. 
HOLBROOK, M.B.; HIRSCHMAN, E.C. The experimental aspects of consumption: consumer fantasies, feelings and fun. Journal of Consumer Research, v. 9, n. 2, p. 132-140, 1982.

JOHNSON, M.D.; ANDERSON, E.W.; FORNELL, C. Rational and adaptive performance expectations in a customer satisfaction framework. Journal of Consumer Research, v. 21, n. 4, p. 695-707, 1995.

KARSAKLIAN, E. Comportamento do consumidor. São Paulo: Atlas, 2009.

KIELING, A.P.; BREI, V.A.; VIEIRA, V.A. A influência da surpresa negativa na adaptação hedônica. Brazilian Business Review, v. 13, n. 3, p. 116-138, 2016.

KOTLER, P.; ARMSTRONG, G. Princípios de marketing. São Paulo: Prentice Hall, 2015.

KOTLER, P.; KELLER, K. L. Administração de marketing. São Paulo: Pearson Prentice Hall, 2012.

LARÁN, J.A.; ROSSI, C.A.V. Surpresa e a formação da satisfação do consumidor. RAE eletrônica UFRGS, v. 5, n. 1, Art. 1, 2006.

LAROS, F. J. M.; STEENKAMP, J. B. E. M. Emotions in consumer behavior: A hierarchical approach. Journal of Business Research, v. 58, n.10, p. 1437-1445, 2005

LOURO, M.J.; PIETERS, R.; ZEELENBERG, M. Negative returns on positive emotions: the influence of pride and self-regulatory goals on repurchase decisions. Journal of Consumer Research, v. 31, n. 4, p. 833-840, 2005.

LUPATINI, M. P. As Transformações Produtivas na Indústria Têxtil-Vestuário e seus Impactos sobre a Distribuição Territorial da Produção e a Divisão do Trabalho Industrial. (Dissertação de Mestrado). Instituto de Economia, Universidade Estadual de Campinas. Campinas, São Paulo, 2004.

MALHOTRA, N. K. Pesquisa de marketing: uma orientação aplicada. 7. ed. Porto Alegre: Bookman, 2012.

MANZINI, E. E VEZZOLI, C. O desenvolvimento de produtos sustentáveis: os requisitos ambientais dos produtos industriais, São Paulo: Editora da Universidade de São Paulo, 2002.

MONTELONGO, A. A influência dos valores humanos na satisfação do consumidor quando da ocorrência de uma surpresa positiva. Dissertação (Mestrado em administração) PPG - Escola de Administração, Universidade Federal do Rio Grande do Sul, Porto Alegre, RS, 2014. 
MÜLLER, M.C. Moda sustentável, consumo consciente e comunicação: estudos de casos no Rio Grande do Sul. Dissertação (Mestrado) Universidade Fernando Pessoa. Porto, 2016.

REFOSCO, E.; et al. O novo consumidor de moda e sustentabilidade. In: VII Colóquio de Moda. Portugal: UMINHO, 2011.

RICHERS, R. O enigmático mais indispensável consumidor: teoria e prática. Revista da Administração, jul./set. 1984.

RICHINS, M.L. Measuring Emotions in the Consumption Experience. Journal of Consumer Research, v. 24, p. 127-146, 1997.

ROSSI, C. A. V.; SLONGO, L.A. Pesquisa de satisfação de clientes: o estado-da-arte e proposição de um método brasileiro. In: ENCONTRO ANUAL DA ASSOCIAÇÃO NACIONAL DOS PROGRAMAS DE PÓS-GRADUAÇÃO EM ADMINISTRAÇÃO, 21.1997, Rio das Pedras. Anais. Rio das Pedras: ANPAD, 1997.

SAMARA, B. S.; MORSCH, M. A. Comportamento do consumidor: conceitos e casos. São Paulo: Pearson Hall, 2005.

SCHIFFMAN, L. G.; KANUK, L. L. Comportamento do consumidor. 6. ed. Rio de Janeiro: LTC, 2000.

SCHULTE, N. K.; LOPEZ, L.D. Sustentabilidade ambiental no produto de moda. In: ENCONTRO DE SUSTENTABILIDADE EM PROJETO DO VALE DO ITAJAÍ, Balneário Camboriú. Anais ENSUS, 2007.

SHETH, J.; MITTAL, B.; NEWMAN, B. Comportamento do Cliente: indo além do comportamento do consumidor, São Paulo: Atlas, 2001.

SIMONSON, I.; TVERSKY, A. Choice in context: tradeoff contrast and extremeness aversion. Journal of Marketing Research, v. 29, p. 281-295, 1992.

SOLOMON, M.R. O Comportamento do Consumidor: comprando, possuindo e sendo, 11 ed. Porto Alegre: Bookman, 2016.

TODESCHINI, B. V. et al. Innovative and sustainable business models in the fashion industry: Entrepreneurial drivers, opportunities, and challenges. Business Horizons, v. 60, n. 6, p.759-770, nov. 2017.

VANHAMME, J. L'influence de la surprise sur la satisfaction des consommateurs étude exploratoire par journal de bord. Recherche et Applications en Marketing, $v$. 16, n. 2, p. 1-31, 2001.

FLETCHER, K. Slow fashion: an invitation for systems change. Fashion Practice, v. 2, p. 259-265, 2010.

R. gest. sust. ambient., Florianópolis, v. 9, n. esp, p. 44-64, mai. 2020. 
WESTBROOK, R. A.; OLIVER, R. L. The dimensionality of consumption emotion patterns and consumer satisfaction. Journal of Consumer Research, v. 18, p. 84-91, 1991.

BLACK, J. S.; GREGERSEN H. B. Expectations, satisfaction, and intention to leave of American expatriate managers in Japan. International Journal of Intercultural Relations, v. 14, p. 485-506, 1990.

BAGOZZI, R. P.; GOPINATH, M.; NYER, P. U. The role of emotions in marketing. Journal of the Academy of Marketing Science, v. 27, n. 2, p. 184-206, 1999. 